\title{
Encapsulated Single Crystal Growth and Annealing of the High-Temperature Superconductor Tl-2201
}

\author{
D.C. Peets ${ }^{\mathrm{a}, *, 1}$, Ruixing Liang ${ }^{\mathrm{a}, \mathrm{c}}$, Mati Raudsepp ${ }^{\mathrm{b}}$, W.N. Hardy ${ }^{\mathrm{a}, \mathrm{c}}$, D.A. Bonn ${ }^{\mathrm{a}, \mathrm{c}, 2}$ \\ ${ }^{a}$ University of British Columbia, Department of Physics \&6 Astronomy, 6224 Agricultural Road, Vancouver, BC, Canada V6T $1 Z 1$ \\ ${ }^{b}$ University of British Columbia, Department of Earth 83 Ocean Sciences, 6339 Stores Road, Vancouver, BC, Canada V6T 1Z4 \\ ${ }^{c}$ Canadian Institute for Advanced Research, Canada
}

\begin{abstract}
Highly-perfect platelet single crystals of $\mathrm{Tl}_{2} \mathrm{Ba}_{2} \mathrm{CuO}_{6+\delta}$ (Tl-2201) were grown by a self-flux technique. A novel 'encapsulation scheme allowed the precursors to react prior to the sealing required to contain volatile thallium oxides, and permitted the removal of melt at the conclusion of growth, reproducibly producing high yields of clean crystals. The crystals were annealed under well-controlled oxygen partial pressures, then characterised. They have sharp superconducting transitions, narrow X-ray rocking curves and a low $4 \%$ substitution of thallium by copper, all evidence of 'their high perfection and homogeneity. The crystals are orthorhombic at most dopings, and a previously unreported commensurate superlattice distortion is observed.
\end{abstract}

Key words:

A1. Crystal structure, A1. X-ray diffraction, A2. Growth from melt, A2. Single crystal growth, B1. Cuprates, B2.

Oxide superconducting materials

PACS: 81.10.Fq, 74.72.Jt, 81.40.Ef, 61.05.cp, 74.25.Ha

\section{Introduction}

In condensed matter physics, a great deal of effort is being applied to the problem of correlated electron systems, and particularly high-temperature superconductivity (HTSC) in the cuprates. Because these superconductors are highly anisotropic and because line nodes in the superconducting energy gap make them highly susceptible to impurities, accurate measurements of their electronic properties rely crucially on the availability of highly perfect, high-purity single crystals.

In the cuprates, hole doping accesses a progression of unusual electronic phases; however, at high doping levels (above where the critical temperature $T_{c}$ peaks) there is evidence for a return to the relative normalcy of Fermi liquid theory, including a resistivity approaching $T^{2}[1]$ and a Fermi surface resembling that predicted by band structure calculations [2, 3, 4]. Despite the tantalising prospect of a phase that can be readily understood, this "overdoped' regime where $T_{c}$ falls back to zero has received significantly less attention than lower doping ranges, due largely to a dearth of suitable samples.

\footnotetext{
* Corresponding author

Email addresses: dpeets@scphys.kyoto-u.ac.jp (D.C. Peets), bonn@physics.ubc.ca (D.A. Bonn)

${ }^{1}$ Current address: Department of Physics, Graduate School of Science, Kyoto University, Kyoto 606-8502, Japan, Tel: +81-75-7533744, FAX: +81-75-753-3783

${ }^{2}$ Requests for samples should be directed to D.A. Bonn.
}

One particularly promising overdoped material is $\mathrm{Tl}_{2} \mathrm{Ba}_{2} \mathrm{CuO}_{6+\delta}$ (Tl-2201) [5], which has a particularly flat $\mathrm{CuO}_{2}$ plane, a relatively simple crystal structure containing no $\left(\mathrm{CuO}_{2}\right)_{n}$ multilayers or $\mathrm{CuO}$ chain layers, and can be overdoped to $T_{c}=0$. Besides being suitable for bulk transport measurements, Tl-2201 has a non-polar cleavage plane within its $\mathrm{Tl}_{2} \mathrm{O}_{2}$ double layer that ensures that surface-sensitive single-particle spectroscopies provide information characteristic of the bulk. Indeed, Tl2201 recently became the first HTSC cuprate on which bulk and surface measurements agreed quantitatively on the same physical property - the Fermi surfaces measured via angular magnetoresistance oscillations (AMRO) [2] and angle-resolved photoemission spectroscopy [3, 4] (ARPES). The excellent agreement indicated that Tl-2201 may be ideal for finally joining the modern single-particle spectroscopies with a host of well-established bulk probes.

Crystals of Tl-2201 have been grown previously by selfflux [6, [7, 8] and $\mathrm{KCl}$ flux 9] techniques. The chief complication in this system is the formation of monovalent $\mathrm{Tl}_{2} \mathrm{O}$, which has a vapour pressure around $0.2-0.3 \mathrm{~atm}$ at the growth temperature [10]. To avoid loss of $\mathrm{Tl}_{2} \mathrm{O}$ the system must be sealed; however, the conversion of $\mathrm{Tl}_{2} \mathrm{O}_{3}$ to $\mathrm{Tl}_{2} \mathrm{O}$ also produces oxygen, which leads to high system pressure if not exhausted. While most groups attempted to limit thallium loss by impeding diffusion, only Hasegawa [8] reported an encapsulation scheme to prevent loss of thallium. In situ separation of crystals from high-temperature melt has not been reported; crystals were typically sepa- 
rated mechanically from cooled, solidified flux, leading to low crystal yields. In the case of $\mathrm{KCl}$ flux growth, the separation is relatively straightforward, but the crystals produced are quite small and there is a risk of potassium contamination.

Tl-2201's hole doping (and thus its $T_{c}$ ) is controlled via variable occupancy of an oxygen interstitial between the Tl-O layers. Setting the oxygen content to desired levels requires post-annealing of the crystals at controlled temperatures and oxygen partial pressures. The vapour pressure of $\mathrm{Tl}$ at elevated temperatures makes proper annealing procedures essential to obtain high-quality crystals, because loss of $\mathrm{Tl}$ means degradation and decomposition of the crystals [11]. Here we report novel processes we have developed for self-flux growth and post-growth annealing of Tl-2201 single crystals.

\section{Single Crystal Growth and Annealing}

Single crystals of Tl-2201 were grown by a copper-rich self flux method in encapsulated crucibles. Precursor powders of $\mathrm{CuO}$ (Alfa Aesar, 99.995\%), $\mathrm{BaO}_{2}$ (Aldrich, 95\%, major impurity $\mathrm{BaCO}_{3}$, heavy metals 0.01\%, 50 ppm Fe) and $\mathrm{Tl}_{2} \mathrm{O}_{3}$ (Aldrich, 99.99\%) were intimately mixed with the cation ratio $\mathrm{Tl}: \mathrm{Ba}: \mathrm{Cu}=2.2: 2: 1.8$, then packed into an alumina crucible containing an upright gold sieve. Barium carbonate was not used because its decomposition to $\mathrm{BaO}$ is very slow at this system's liquidus temperature.

The growth technique is depicted in Fig. 1. A gold lid was affixed to the crucible, then the crucible and lid were held between two alumina rams in a tube furnace inclined $20^{\circ}$ and supporting a transverse temperature gradient. Sufficient pressure was applied to the rams to hold the crucible in place, but not to seal it, while the crucible was heated and the precursors reacted. This acted as a barrier to the loss of thallium by diffusion while allowing the release of any overpressure due to evolved oxygen. After soaking at $935^{\circ} \mathrm{C}$ (above the liquidus) for two hours to allow evolving gas to exhaust, the pressure applied to the gold lid was increased to several atmospheres, sealing the crucible. Evolved vapours were swept from the furnace by flowing oxygen, then bubbled through acid to remove any thallium.

The melt's temperature was reduced at a rate of $0.3^{\circ} \mathrm{C} / \mathrm{h}$ along the liquidus curve to $890^{\circ} \mathrm{C}$, just above the Tl-2201 - CuO eutectic, which was found to be slightly lower than previously reported [12]. At this temperature, the crystals were separated from the remaining melt by rotating the rams and crucible by $180^{\circ}$; the gold sieve ensured that the crystals remained clear of the melt. The temperature was reduced to $825^{\circ} \mathrm{C}$ for a 48 hour anneal to make the distribution of substituted copper atoms more homogeneous, followed by free cooling to room temperature.

The charge's mass was measured before and after growth; the mass lost was typically less than that expected assuming the loss of one oxygen atom from $\mathrm{BaO}_{2}$ and two

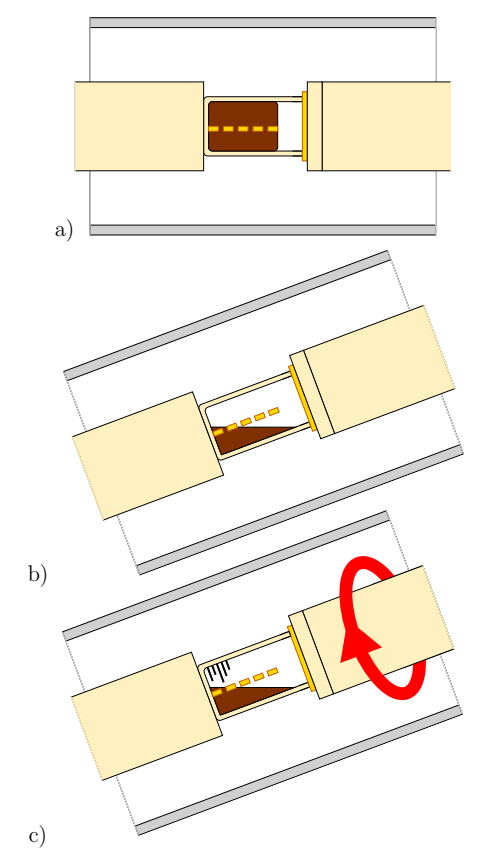

Figure 1: (Colour online) Schematic depiction of the in situ flux separation scheme. a) The filled crucible and gold lid are inserted between two alumina rams; the crucible contains an upright gold sieve. b) The furnace is inclined, the charge melted, and crystals are grown on slow cooling. c) The rams are rotated, separating the crystals from excess flux with the aid of the gold sieve.

from $\mathrm{Tl}_{2} \mathrm{O}_{3}$. Since $\mathrm{BaO}_{2}$ decomposes around $800^{\circ} \mathrm{C}$ and $\mathrm{Tl}_{2} \mathrm{O}_{3}$ at higher temperatures, an upper limit of $5 \%$ may be obtained for the thallium lost if it is assumed that all thallium remains trivalent and all mass losses beyond the decomposition of $\mathrm{BaO}_{2}$ are attributable to loss of thallium oxide. Hasegawa et al. [8] pre-reacted the precursors below the eutectic temperature prior to encapsulating the crucible, but some crucibles still ruptured from the pressure, suggesting gases are evolved when the precursors melt. Here, the crucible was encapsulated after the melting of the precursors. This avoids eruption of the crucible contents and allows for more reliable sealing, but at the cost of a few percent of the thallium.

Irregularly shaped black, platelet single crystals typically $1 \times 1 \times 0.01 \mathrm{~mm}^{2}$ in size with mirror surfaces were grown reproducibly by this method. After mechanical separation from the crucible, they were annealed under controlled oxygen partial pressures at temperatures between $290^{\circ} \mathrm{C}$ and $500^{\circ} \mathrm{C}$, producing sharp superconducting transitions at temperatures ranging from 5 to $85 \mathrm{~K}$. No attempts to reach optimal doping (corresponding to at least $T_{c}=93 \mathrm{~K} \mathrm{[13]}$ ) were made, to avoid surface decomposition, but some batches exhibited as-grown $T_{c} \mathrm{~s}$ as high as $90 \mathrm{~K}$. The $T_{c}$ produced by the oxygen partial pressure and anneal temperature resembled those reported previously on ceramics [14].

Two oxygen-annealing schemes are depicted in Fig. 2 . To access the strongly overdoped regime (high oxygen con- 
tents), corresponding to $T_{c} \mathrm{~s}$ below $\sim 45 \mathrm{~K}$, oxygen partial pressures of $10^{-4}$ to 1 atm were applied via flowing mixtures of oxygen and nitrogen gases. Within this oxygen partial pressure range, the loss of thallium from the crystals remained undetectable even after anneals of several weeks.

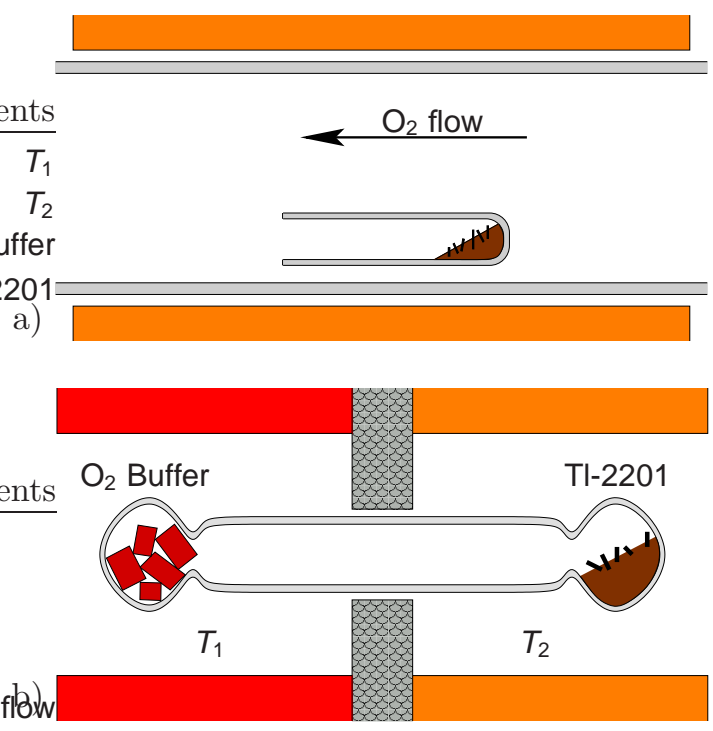

Figure 2: (Colour online) Annealing schemes used to obtain a) strongly overdoped and b) moderately overdoped crystals. In a), the crystals are placed on Tl-2201 powder in flowing oxygen or an appropriate mixed gas; in b), the Tl-2201 powder serves as a thallium buffer, an oxygen partial pressure buffer is situated at the other end of the quartz ampoule and the two ends' temperatures may be controlled separately for independent control over the oxygen partial pressure and the anneal temperature.

To access the lower doping regime, corresponding to $T_{c} \mathrm{~s}$ above $\sim 45 \mathrm{~K}$, annealing at oxygen partial pressures lower than $10^{-4} \mathrm{~atm}$ is required [14]. At these low oxygen pressures, the loss of thallium from the crystals becomes an issue and the annealing must be carried out in an enclosed environment. As shown in Fig. 2(b), Tl-2201 crystals embedded in Tl-2201 powder were placed in one end of a sealed quartz capsule and an oxygen buffer consisting of a mixture of $\mathrm{CuO}$ and $\mathrm{Cu}_{2} \mathrm{O}$ at the other end. The two ends were thermally isolated by fibrous ceramic insulation so that the temperatures of the crystals and the buffer could be controlled independently. The Tl-2201 powder releases sufficient $\mathrm{Tl}_{2} \mathrm{O}$ to produce the required equilibrium $\mathrm{Tl}_{2} \mathrm{O}$ partial pressure in the capsule, essentially eliminating the loss of $\mathrm{Tl}$ from the crystals. The oxygen partial pressure is controlled via the temperature of the buffer, through the chemical equilibrium between $\mathrm{CuO}$ and $\mathrm{Cu}_{2} \mathrm{O}$.

At the conclusion of each anneal, the crystals were quenched by plunging the quartz tube or capsule into an icewater bath. This preserves the equilibrium oxygen content established under the annealing conditions and ensures a homogeneous dopant distribution.

\section{Characterisation}

X-ray rocking curves of several Tl-2201 crystals were collected on a Philips X'Pert Pro single crystal X-ray diffractometer, using a copper $K \alpha_{1}$ vertical line source excited by $45 \mathrm{kV}$ and $40 \mathrm{~mA}$. Diffracted X-rays were detected using a serial detector with no scanning slit. Each $\sim 1 \mathrm{~mm}^{2}$ crystal was fully illuminated. Rocking curve widths $(\mathrm{FWHM})$ were $0.025^{\circ} \sim 0.050^{\circ}$, indicating a high degree of crystalline perfection - comparable to good $\mathrm{YBa}_{2} \mathrm{Cu}_{3} \mathrm{O}_{6+\delta}$ grown in zirconia crucibles. A typical (ll 0 10) rocking curve is shown in Fig. 5)(a).

Electron-probe micro-analyses (EPMA) of several crystals were performed using a fully-automated CAMECA SX-50 instrument in wavelength-dispersion mode. EPMA was performed using an excitation voltage of $15 \mathrm{kV}$, a beam current of $20 \mathrm{nA}$, peak and background count times of 80 and $40 \mathrm{~s}$ respectively, and a spot diameter of $10 \mu \mathrm{m}$; initial data reduction employed the "PAP" $\varphi(\rho Z)$ method [15]. For the cations analyzed, the following standards, $\mathrm{X}$-ray lines and monochromator crystals were used: elemental Tl, TlM $\alpha$, PET; $\mathrm{YBa}_{2} \mathrm{Cu}_{3} \mathrm{O}_{6.920}, \mathrm{BaL} \alpha$, PET; and $\mathrm{YBa}_{2} \mathrm{Cu}_{3} \mathrm{O}_{6.920}, \mathrm{CuK} \alpha$, LIF. Tight Pulse Height Analysis (PHA) control was used to eliminate to the degree possible any interference from higher-order lines.

Crystals were epoxied to the face of an acrylic disc; care was taken to ensure the surface remained parallel to the disc, and 10-15 flat sites were studied per crystal, then averaged. No significant variations were seen among sites on the same crystal or among crystals from similar growth conditions, but variations were observed among crystals grown using different initial cation ratios. Because the barium results were less consistent, possibly due to surface topography, the cation composition was normalised to $(\mathrm{Tl}+\mathrm{Cu})=3-$ in Tl-2201, $\mathrm{Cu}$ is known to substitute for $\mathrm{Tl}$ and no cation vacancies exist. The cation composition was determined to be $\mathrm{Tl}_{1.920(2)} \mathrm{Ba}_{1.96(2)} \mathrm{Cu}_{1.080(2)} \mathrm{O}_{6+\delta}(2 \sigma$ uncertainties), indicating that $4 \%$ of $\mathrm{Tl}$ is substituted by $\mathrm{Cu}$, slightly lower than other reported results [16, 6, 17], including the $5 \%$ substitution in the author's earlier generation of crystals [4]. Better encapsulation, and hence lower loss of $\mathrm{Tl}$ during the growth, may have contributed to the lower level of $\mathrm{Cu}$ substitution for $\mathrm{Tl}$. A separate check for aluminum contamination from the crucible excluded it at the $50 \mathrm{ppm}$ level; it was not possible to check for gold contamination, but this is expected to be minimal due to the absence of sites like YBCO's $\mathrm{CuO}$ chains that are known to accommodate $\mathrm{Au}^{1+}$.

The crystals' transition temperatures $T_{c}$ and widths $\Delta T_{c}$ were characterised using a Quantum Design MPMS SQUID magnetometer; fields $H \| c$ of only $1 \sim 2$ Oe were employed to minimise broadening. Normalised field-cooled SQUID magnetisation curves for Tl-2201 crystals at a variety of dopings are shown in Fig. 3. Transition widths of $1 \sim 2 \mathrm{~K}$, indicative of homogeneous doping, can be obtained throughout the doping range from $18 \mathrm{~K}$ to $75 \mathrm{~K}$. These transitions are narrower than those reported previ- 
ously on Tl-2201, and compare well with crystals of other cuprates. The Meissner fraction (the fraction of the magnetic flux excluded when cooling through $T_{c}$ ) was typically $40 \sim 60 \%$, which compares well with field-cooled results on high-quality crystals of other cuprates.

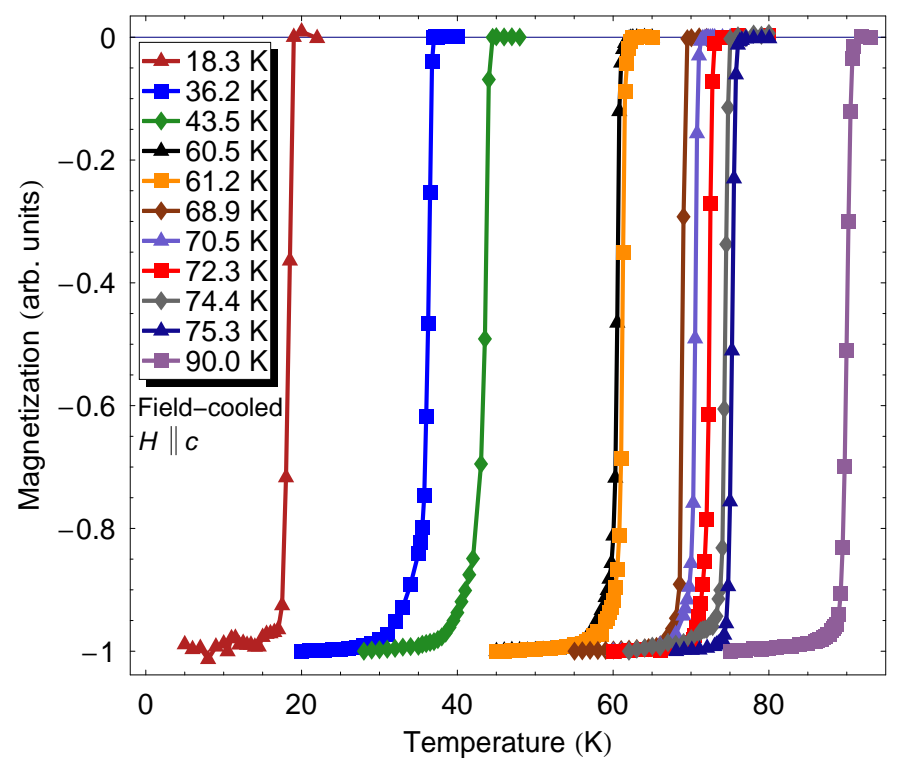

Figure 3: (Colour online) Field-cooled magnetisation curves of several Tl-2201 crystals. The $T_{c}=90 \mathrm{~K}$ crystal was as grown; the others were annealed. Transition widths $(10 \%-90 \%)$ are typically $0.75 \sim 1.5 \mathrm{~K}$ - the crystals may be homogeneously doped over a wide doping range. Data were collected in fields $H \| c$ of $1 \sim 2$ Oe.

Table 1 compares the results of previous single crystal studies with the abovementioned techniques used for thallium containment and annealing, selected transition temperature widths $\Delta T_{c}$, and several crystal structure results discussed below. The transition widths shown in Fig. 3 compare well with previous results, indicating the high quality and homogeneity of the crystals.

\section{Crystal Structure}

X-ray and neutron powder diffraction studies on Tl2201 have revealed the existence of two distinguishable phases of Tl-2201, with orthorhombic and tetragonal symmetry [19, 20]. Both the degree of Cu substitution on the $\mathrm{Tl}$ site and the amount of interstitial oxygen between the Tl-O layers control the structure 18, 6, 17, 21, 22, 13. Oxygen vacancies in the $\mathrm{Tl}-\mathrm{O}$ layers have also been observed for samples with low oxygen contents (near optimal doping). The most thorough structure investigation was by Wagner et al. 13], which elucidated the interplay between oxygen interstitials, oxygen vacancies, cation substitution, $T_{c}$, and orthorhombicity. Structure refinement by single crystal diffraction should provide more structural details, but so far single crystal X-ray diffraction [6, 17] has only been carried out on tetragonal crystals; the high concentration of $\mathrm{Cu}$ substituents and low occupancy of interstitials may make some structural features difficult to observe. Measurements of the lattice parameters on the author's earlier crystals showed evidence of orthorhombic symmetry [4], not previously reported in crystals. Since the orthorhombicity was enhanced at high oxygen contents, the choice of near-optimally doped crystals may be responsible for previous studies' non-observation of orthorhombicity. However, earlier work has equated orthorhombicity with stoichiometric, non-superconducting Tl-2201 [23, 20], suggestive of a link between symmetry and superconductivity, and orthorhombicity has only been reported in one crystal. Here we provide confirmation that superconducting crystals may be orthorhombic as well as tetragonal.

Lattice parameters were measured on several dopings of Tl-2201 crystal at $-100^{\circ} \mathrm{C}$ on a Bruker X8 Apex diffractometer, using a CCD area detector and a molybdenum $\mathrm{X}$-ray source. A full X-ray structure refinement was performed on a crystal with $T_{c}=75 \mathrm{~K}$, using 3319 reflections (312 unique) in the $k$-space region $-7 \leq h \leq 7$, $-7 \leq k \leq 7,-31 \leq l \leq 32$, by a full-matrix least-squares minimization of $F^{2}$. Structure refinement was performed using SHELXL-97 24], in the orthorhombic space group Fmmm for generality; this unit cell is rotated $45^{\circ}$ relative to the tetragonal cell and contains four formula units. All atoms were constrained to their symmetric positions (relaxing this constraint led to only minor improvements), no sites were split, and occupancies of all sites except $\mathrm{O}(4)$ were fixed at full occupancy; atoms other than $\mathrm{O}(4)$ were allowed anisotropic thermal parameters. Besides refining for occupancy of the $\mathrm{O}(4)$ site, a refinement was performed for copper substitution on the thallium site. Note that since all previously published data were collected at room temperature, thermal expansion must be taken into account when comparing these data to published values.

The crystals' lattice parameters are summarised in Table 2 orthorhombicity is characterised using the orthorhombic strain $\eta=2(b-a) /(b+a)$ as a figure of merit. The $T_{c}=9 \mathrm{~K}$ crystal was extracted from an early batch that leaked during growth, likely leading to higher cation substitution. Aside from this crystal, more heavily overdoped samples (lower $T_{c} \mathrm{~s}$ ) are more orthorhombic. This is consistent with Wagner's results [13], and constitutes important confirmation of orthorhombicity in superconducting single crystalline Tl-2201 — the symmetry does not determine whether the material superconducts.

The refined atomic positions for the $T_{c}=75 \mathrm{~K}$ crystal are presented in Table 3. the full thermal displacement parameters $U_{i j}$ for these atoms are reproduced in Table 4 and the resulting orthorhombic crystal structure is shown in Fig. 4. Because no sites were split, there were few refinable parameters. The substitution of copper atoms onto the thallium site was refined with the site constrained to full occupancy, yielding the substitution level $\mathrm{Tl}_{1.914(14)} \mathrm{Cu}_{0.086(14)}$, in excellent agreement with the EPMA result. The O(4) occupancy was also refined, but 
Table 1: Comparison to literature: the preparation and properties of the crystals reported in this work are compared with selections from previous reports on single-crystalline Tl-2201. Included are containment method and annealing performed; $T_{c}$ with its width $\Delta T_{c}$ reported as an uncertainty in the last digit; cation substitution level $z$ and oxygen content $6+\delta$ in $\left(\mathrm{Tl}_{1-z} \mathrm{Cu}_{z}\right)_{2} \mathrm{Ba}_{2} \mathrm{CuO} 6+\delta$ by EPMA and $\mathrm{X}-\mathrm{ray}$ diffraction; and space group. A dash indicates properties that were not reported; crystals on which X-ray refinement was not performed are denoted "tetragonal" and "orthorhombic" in place of a space group. The crystal reported in Ref. [17] was grown in the presence of calcium.

\begin{tabular}{|c|c|c|c|c|c|c|c|}
\hline Source & Sealing & Annealing & $T_{c}\left(\Delta T_{c}\right)$ & $z(\mathrm{EPMA})$ & $z$ (X-ray) & $6+\delta$ (X-ray) & Symmetry \\
\hline \multirow[t]{4}{*}{ "This work } & \multirow{4}{*}{$\begin{array}{c}\text { Au lid, } \\
\text { variable } \\
\text { pressure }\end{array}$} & \multirow{4}{*}{$\begin{array}{c}\text { Cation, } \\
P_{\mathrm{O}_{2}} / P_{\mathrm{Tl}_{2} \mathrm{O}}\end{array}$} & $\overline{900(1) \mathrm{K}}$ & - & - & - & Tetragonal \\
\hline & & & $75(1) \mathrm{K}$ & 0.040 & 0.043 & $6.29(18)$ & Fmmm \\
\hline & & & $55(2) \mathrm{K}$ & 0.040 & - & - & Orthorhombic \\
\hline & & & $9(5) \mathrm{K}$ & - & - & - & Orthorhombic \\
\hline Ref. [18] & Au tube & - & $90(7) \mathrm{K}$ & - & - & - & $\mathrm{I} 4 / \mathrm{mmm}$ \\
\hline Ref. [6] & $\mathrm{Au}$ lid & $\begin{array}{l}P_{\mathrm{O}_{2}}, \\
\text { see [1] }\end{array}$ & $12.4(8) \mathrm{K}$ & 0.075 & 0.068 & 6.0 & $\mathrm{I} 4 / \mathrm{mmm}$ \\
\hline Ref. [17] & Alunde anvil & 二 & $110(15) \mathrm{K}$ & 0.05 & 0.073 & 6.0 & $\mathrm{I} 4 / \mathrm{mmm}$ \\
\hline Ref. [7] & $\begin{array}{c}\text { Multilayered } \\
\text { crucible }\end{array}$ & - & $30(-) \mathrm{K}$ & - & 0.050 & $6.18(4)$ & $\mathrm{I} 4 / \mathrm{mmm}$ \\
\hline Ref. [8] & $\begin{array}{l}\text { Au capsule, } \\
\mathrm{Al}_{2} \mathrm{O}_{3} \text { bomb }\end{array}$ & $\begin{array}{c}P_{\mathrm{O}_{2}} \\
\text { in situ }\end{array}$ & $25(3) \mathrm{K}$ & $\begin{array}{l}\text { Vacancy: } \\
0.05\end{array}$ & - & - & $\mathrm{I} 4 / \mathrm{mmm}$ \\
\hline \multirow[t]{2}{*}{ Ref. [4] } & \multirow{2}{*}{$\begin{array}{l}\text { Weighted } \\
\text { Au lid }\end{array}$} & \multirow{2}{*}{$\begin{array}{c}P_{\mathrm{O}_{2}}, \text { no } \\
\text { details }\end{array}$} & $24(4) \mathrm{K}$ & 0.055 & - & - & Orthorhombic \\
\hline & & & $67.7(7) \mathrm{K}$ & 0.055 & - & - & Tetragonal \\
\hline
\end{tabular}

Table 2: Lattice parameters and orthorhombic strain $\eta$ for four dopings of Tl-2201 as determined by single-crystal X-ray diffraction; uncertainties are $1 \sigma$. The $T_{c}=9 \mathrm{~K}$ crystal is thought to have higher cation substitution.

\begin{tabular}{|r|l|l|c|c|}
\hline$T_{c}$ & \multicolumn{1}{|c|}{$a(\AA)$} & \multicolumn{1}{c|}{$b(\AA)$} & $c(\AA)$ & $\eta(\% \circ)$ \\
\hline \hline $90 \mathrm{~K}$ & $5.4603(14)$ & $5.4602(13)$ & $23.1901(59)$ & $0.0(4)$ \\
\hline $75 \mathrm{~K}$ & $5.4477(9)$ & $5.4484(9)$ & $23.1711(35)$ & $0.13(23)$ \\
\hline $55 \mathrm{~K}$ & $5.4424(12)$ & $5.4550(12)$ & $23.1428(51)$ & $2.31(31)$ \\
\hline $9 \mathrm{~K}$ & $5.4067(15)$ & $5.4109(14)$ & $22.9219(54)$ & $0.77(35)$ \\
\hline
\end{tabular}

the low sensitivity of this technique to light atoms and the low occupancy of the site at this doping led to an occupancy consistent with zero to within $2 \sigma-\mathrm{O}_{0.29(18)}$ - and the site departed from its previously reported position. The refinement's $R_{1}$ factor was $2.35 \% ; w R_{2}$ was $5.68 \%$ on all data. The cation substitution and $\mathrm{O}(4)$ occupancy results, along with the symmetry, are compared against previous reports on single crystals in Table 1.

It is evident from the crystal structure in Fig. 4 and the atomic displacement parameters in Table 4 that the refinement fails to fully capture the structure in the $\mathrm{Ba}-\mathrm{O}$ and $\mathrm{Tl}-\mathrm{O}$ layers. This can be dealt with by splitting the $\mathrm{Tl}$ and $\mathrm{O}(3)$ sites [23, 16, 17, 13], which provides limited information about which directions each site departs from its symmetric position, while some studies have identified satellite peaks corresponding to an incommensurate superlattice modulation in the TlO layers [25, 20, 22, . Simulated precession photographs were generated from the diffraction data, to help clarify the crystal structure. The $(h k 0)$ plane for the $T_{c}=75 \mathrm{~K}$ crystal is shown in Fig. [5)(b). Weak
Table 3: Refined atomic positions and equivalent isotropic thermal displacement parameter $U_{e q}$, defined as one third of the trace of the orthogonalised $U_{i j}$ tensor, for the $T_{c}=75 \mathrm{~K}$ crystal. See Table 4 for the $U_{i j}$ parameters and Fig. 4 for the crystal structure.

\begin{tabular}{|l|l|l|l|l|}
\hline Atom & $x / a$ & $y / b$ & \multicolumn{1}{|c|}{$z / c$} & $U_{e q}$ \\
\hline \hline $\mathrm{Tl}$ & 0.0000 & 0.0000 & $0.29732(2)$ & $0.0108(2)$ \\
\hline $\mathrm{Ba}$ & 0.0000 & 0.0000 & $0.08306(3)$ & $0.0028(3)$ \\
\hline $\mathrm{Cu}$ & 0.0000 & 0.0000 & 0.5000 & $0.0023(4)$ \\
\hline $\mathrm{O}(1)$ & 0.2500 & 0.2500 & 0.0000 & $0.0043(13)$ \\
\hline $\mathrm{O}(2)$ & 0.0000 & 0.0000 & $0.3833(4)$ & $0.0087(16)$ \\
\hline $\mathrm{O}(3)$ & 0.0000 & 0.0000 & $0.2112(5)$ & $0.055(6)$ \\
\hline $\mathrm{O}(4)$ & 0.2500 & 0.2500 & $0.283(14)$ & $0.14(15)$ \\
\hline
\end{tabular}

Table 4: Anisotropic displacement parameters for those atomic positions in Table 3 which were refined assuming an anisotropic electron density. $U_{23}$ and $U_{13}$, zero by symmetry, are excluded from this table. Atoms in the $\mathrm{Tl}-\mathrm{O}$ and $\mathrm{Ba}-\mathrm{O}$ layers are particularly anisotropic, suggesting this to be the location of the superlattice modulation.

\begin{tabular}{|l|l|l|l|c|}
\hline Atom & \multicolumn{1}{|c|}{$U_{11}$} & \multicolumn{1}{|c|}{$U_{22}$} & $U_{33}$ & $U_{12}$ \\
\hline \hline $\mathrm{Tl}$ & $0.0130(3)$ & $0.0190(4)$ & $0.0006(3)$ & 0 \\
\hline $\mathrm{Ba}$ & $0.0000(4)$ & $0.0052(4)$ & $0.0031(4)$ & 0 \\
\hline $\mathrm{Cu}$ & $0.0000(8)$ & $0.0030(8)$ & $0.0040(9)$ & 0 \\
\hline $\mathrm{O}(1)$ & $0.000(3)$ & $0.006(3)$ & $0.007(3)$ & $0.004(2)$ \\
\hline $\mathrm{O}(2)$ & $0.006(4)$ & $0.017(4)$ & $0.003(3)$ & 0 \\
\hline $\mathrm{O}(3)$ & $0.086(15)$ & $0.080(14)$ & $0.000(4)$ & 0 \\
\hline
\end{tabular}




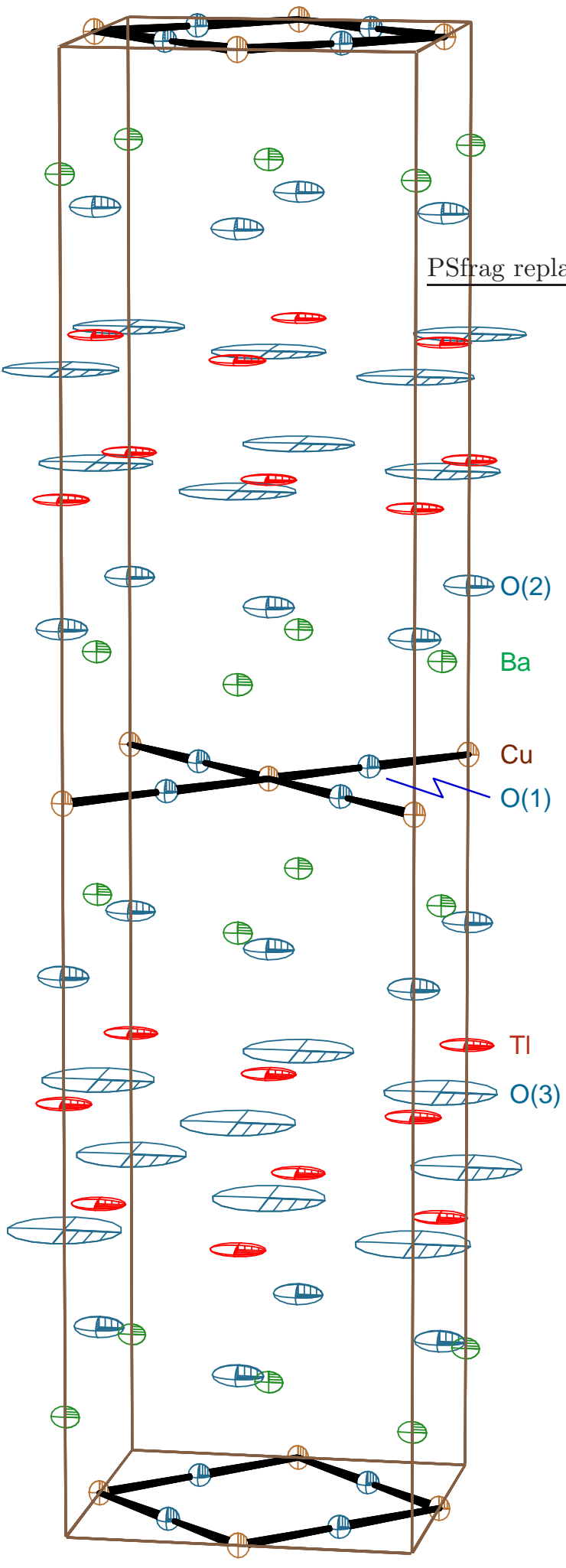

Figure 4: (Colour online) Refined orthorhombic crystal structure with $98 \%$ probability ellipsoids for each atom's nuclear position, $T_{c}=$ $75 \mathrm{~K} \mathrm{Tl}-2201$ crystal, indentifying each site and clearly suggesting that the structure of the Tl-O and $\mathrm{Ba}-\mathrm{O}$ layers is not fully captured by the refinement. The $\mathrm{O}(4)$ interstitial has been excluded for clarity.
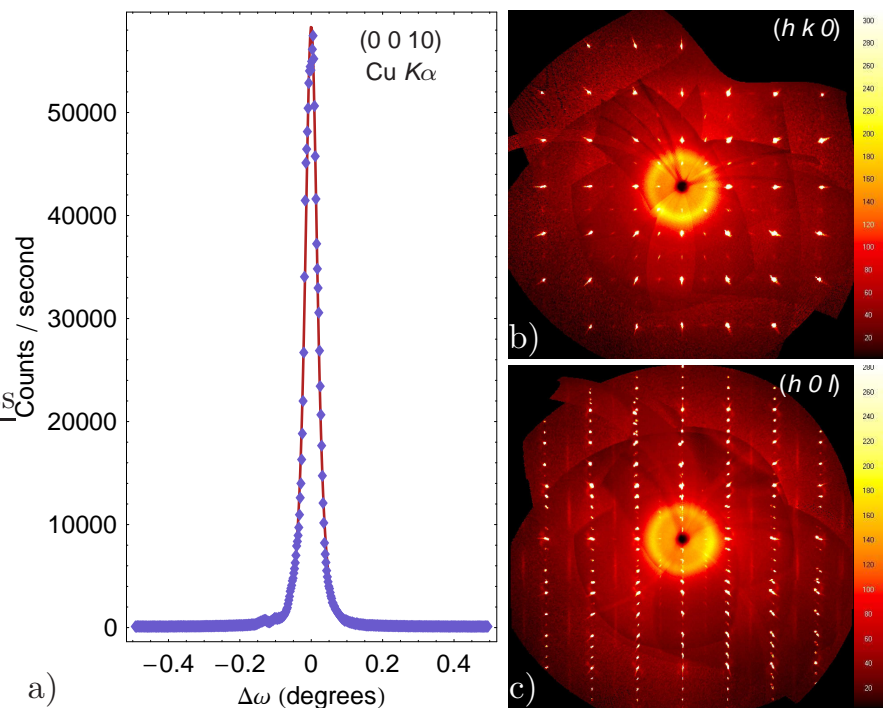

Figure 5: (Colour online) a) $\left(\begin{array}{lll}0 & 0 & 10\end{array}\right) \mathrm{X}$-ray rocking curve, $T_{c}=$ $75 \mathrm{~K}$ crystal. The full width at half-maximum is $0.040^{\circ}$. b) and c) Simulated X-ray precession photos constructed from the single crystal diffraction data, for $(h k 0)$ and $(h 0 l)$ planes respectively, showing weak rods of scattering corresponding to a commensurate superlattice modulation that doubles the unit cell along $a$ and $b$ but is largely uncorrelated along $c$.

diffraction spots correspond to a commensurate superlattice modulation that doubles the in-plane lattice constants. As can be seen from the $(h 0 l)$ plane in Fig. 5(c) (the $(0 k l)$ plane appears similar and is not reproduced here), these more closely resemble rods than points, although they do exhibit some point-like character — the superlattice modulation is not well correlated along the $c$-axis.

It is clear from the thermal displacement parameters that the superlattice modulation involves both the $\mathrm{BaO}$ and $\mathrm{TlO}$ layers, and the diffraction patterns indicate that the modulation is commensurate with the lattice, but the exact nature of the modulation was not determined. That it is commensurate with the lattice is a departure from previously reported superlattice modulations in this material. The doubling of the unit cell along $a$ and $b$ may be important to consider in the interpretation of singleparticle spectroscopic data.

\section{Conclusion}

In summary, single crystals of Tl-2201 were reproducibly grown by a copper-rich self-flux method, employing a novel encapsulation scheme that permits the escape of evolved gases as the precursors react, then seals the crucible for a fully encapsulated growth. The crystals were separated from the molten flux by rotating the crucible and letting the flux flow through a gold sieve. This resulted in much higher yields of clean crystals compared to the mechanical separation of crystals from solidified flux reported in previous work. The crystals were annealed under well-controlled oxygen partial pressures, allowing the 
preparation of crystals throughout a wide range of hole doping and $T_{c}$. In particular, crystals with low oxygen contents (high $T_{c} \mathrm{~s}$ ) were prepared by annealing in an encapsulated environment using a $\mathrm{CuO} / \mathrm{Cu}_{2} \mathrm{O}$ mixture as an oxygen buffer. This avoided the loss of $\mathrm{Tl}$ and surface degradation reported in earlier work.

$\mathrm{X}$-ray rocking curves and magnetisation measurements demonstrated that the crystals had a very high degree of crystalline perfection and that the dopant distribution was homogeneous. The compositions obtained from EPMA and X-ray diffraction on $T_{c}=75 \mathrm{~K}$ crystals, $\left(\mathrm{Tl}_{1.920(2)} \mathrm{Cu}_{0.080(2)}\right) \mathrm{Ba}_{1.96(2)} \mathrm{CuO}_{6+\delta}$ and $\left(\mathrm{Tl}_{1.914(14)} \mathrm{Cu}_{0.086(14)}\right) \mathrm{Ba}_{2} \mathrm{CuO}_{6.29(18)}$ respectively, are closer to stoichiometric than are typically reported on crystals, possibly due to more effective retention of thallium.

The trends in the lattice parameters with doping are consistent with those reported previously [13, 4], and the presence of an orthorhombic distortion in overdoped superconducting crystals is confirmed. A structure refinement indicated the presence of a previously unobserved commensurate superlattice modulation in the $\mathrm{Ba}-\mathrm{O}$ and primarily Tl-O layers, doubling the unit cell along $a$ and $b$, but not well correlated along $c$. Further work will be required to determine the exact nature of this modulation.

\section{Acknowledgements}

This work was supported by the Natural Sciences and Engineering Research Council of Canada (NSERC). The authors are grateful to B. O. Patrick and the UBC Vancouver Chemistry Department's Structural Chemistry Facility for assistance with the collection and analysis of X-ray diffraction data and to A. P. Mackenzie for helpful discussions.

\section{References}

[1] A. P. Mackenzie, S. R. Julian, D. C. Sinclair, C. T. Lin, Normalstate magnetotransport in superconducting $\mathrm{Tl}_{2} \mathrm{Ba}_{2} \mathrm{CuO}_{6+\delta}$ to millikelvin temperatures, Phys. Rev. B 53 (1996) 5848-5855. doi:10.1103/PhysRevB.53.5848

[2] N. E. Hussey, M. Abdel-Jawad, A. Carrington, A. P. Mackenzie, L. Balicas, A coherent three-dimensional Fermi surface in a high-transition-temperature superconductor, Nature 425 (2003) 814-817. doi:10.1038/nature01981

[3] M. Platé, J. D. F. Mottershead, I. S. Elfimov, D. C. Peets, R. Liang, D. A. Bonn, W. N. Hardy, S. Chiuzbaian, M. Falub, M. Shi, L. Patthey, A. Damascelli, Fermi surface and quasiparticle excitations of overdoped $\mathrm{Tl}_{2} \mathrm{Ba}_{2} \mathrm{CuO}_{6+\delta}$, Phys. Rev. Lett. 95 (2005) 077001. doi:10.1103/PhysRevLett.95.077001

[4] D. C. Peets, J. D. F. Mottershead, B. Wu, I. S. Elfimov, R. Liang, W. N. Hardy, D. A. Bonn, M. Raudsepp, N. J. C. Ingle, A. Damascelli, $\mathrm{Tl}_{2} \mathrm{Ba}_{2} \mathrm{CuO}_{6+\delta}$ brings spectroscopic probes deep into the overdoped regime of the high- $T_{c}$ cuprates, New J. Phys. 9 (2007) 28. doi:10.1088/1367-2630/9/2/028

[5] Z. Z. Sheng, A. M. Hermann, Superconductivity in the rareearth-free $\mathrm{Tl}-\mathrm{Ba}-\mathrm{Cu}-\mathrm{O}$ system above liquid-nitrogen temperature, Nature 332 (1988) 55-58. doi:10.1038/332055a0
[6] R. S. Liu, S. D. Hughes, R. J. Angel, T. P. Hackwell, A. P. Mackenzie, P. P. Edwards, Crystal structure and cation stoichiometry of superconducting $\mathrm{Tl}_{2} \mathrm{Ba}_{2} \mathrm{CuO}_{6+\delta}$ single crystals, Physica C 198 (1992) 203-208. doi:10.1016/0921-4534(92) 90192-F

[7] N. N. Kolesnikov, M. P. Kulakov, V. N. Molchanov, I. F. Schegolev, R. P. Shibaeva, V. I. Simonov, R. A. Tamazyan, O. M. Vyasilev, Comparative study of Tl-2201 single crystals with $T_{c}=30$ and $110 \mathrm{~K}$ by means of X-ray structural analysis and NMR, Physica C 242 (1995) 385-392. doi:10.1016/0921-4534(94)02420-0

[8] M. Hasegawa, H. Takei, K. Izawa, Y. Matsuda, Crystal growth techniques for Tl-based cuprate superconductors, J. Cryst. Growth 229 (2001) 401-404. doi:10.1016/S0022-0248(01)01189-7

[9] T. Manako, Y. Kubo, Y. Shimakawa, Transport and structural study of $\mathrm{Tl}_{2} \mathrm{Ba}_{2} \mathrm{CuO}_{6+\delta}$ single crystals prepared by the KCl flux method, Phys. Rev. B 46 (1992) 11019-11024. doi:10.1103/PhysRevB.46.11019

[10] M. P. Siegal, E. L. Venturini, B. Morosin, T. L. Aselage, Synthesis and properties of $\mathrm{Tl}-\mathrm{Ba}-\mathrm{Cu}-\mathrm{O}$ superconductors, J. Mater. Res. 12 (1997) 2825-2854. doi:10.1557/JMR.1997.0378

[11] O. M. Vyaselev, N. N. Kolesnikov, M. P. Kulakov, I. F. Schegolev, Tl NMR study of $\mathrm{Tl}_{2} \mathrm{Ba}_{2} \mathrm{CuO}_{x}$ single crystals with various $T_{c}$, Physica C 199 (1992) 50-58. doi:10.1016/0921-4534(92) 90539-0

[12] J. L. Jorda, T. K. Jondo, R. Abraham, M. T. CohenAdad, C. Opagiste, M. Couach, A. F. Khoder, F. Sibieude, Preparation of pure $\mathrm{Tl}_{2} \mathrm{Ba}_{2} \mathrm{CuO}_{6 \pm x}$ : The contribution of phase equilibrium studies, Physica C 205 (1993) 177-185. doi:10.1016/0921-4534(93) 90185-S

[13] J. L. Wagner, O. Chmaissem, J. D. Jorgensen, D. G. Hinks, P. G. Radaelli, B. A. Hunter, W. R. Jensen, Multiple defects in overdoped $\mathrm{Tl}_{2} \mathrm{Ba}_{2} \mathrm{CuO}_{6+\delta}$ : Effects on structure and superconductivity, Physica C 277 (1997) 170-182. doi:10.1016/S0921-4534(97)00062-2

[14] C. Opagiste, G. Triscone, M. Couach, T. K. Jondo, A. Junod, A. F. Khoder, J. Muller, Phase diagram of the $\mathrm{Tl}_{2} \mathrm{Ba}_{2} \mathrm{CuO}_{6}$ compounds in the $T, p\left(\mathrm{O}_{2}\right)$ plane, Physica C 213 (1993) 17-25. doi:10.1016/0921-4534(93) 90753-D

[15] J. L. Pouchou, F. Pichoir, "PAP" $\varphi(\rho z)$ procedure for improved quantitative microanalysis, in: J. T. Armstrong (Ed.), Microbeam Analysis 1985, San Francisco Press, 1985, pp. 104106.

[16] Y. Shimakawa, Y. Kubo, T. Manako, H. Igarashi, F. Izumi, H. Asano, Neutron-diffraction study of $\mathrm{Tl}_{2} \mathrm{Ba}_{2} \mathrm{CuO}_{6+\delta}$ with various $T_{c}$ 's from 0 to $73 \mathrm{~K}$, Phys. Rev. B 42 (1990) 1016510171. doi:10.1103/PhysRevB.42.10165

[17] N. N. Kolesnikov, V. E. Korotkov, M. P. Kulakov, V. N. Molchanov, R. A. Tamazyan, V. I. Simonov, Structure of superconducting single crystals of 2201 thallium cuprate $\left(\mathrm{Tl}_{1.85} \mathrm{Cu}_{0.15}\right) \mathrm{Ba}_{2} \mathrm{CuO}_{6}, T_{c}=110 \mathrm{~K}$, Physica C 195 (1992) 219-224. doi:10.1016/0921-4534(92) 90343-B

[18] C. C. Torardi, M. A. Subramanian, J. C. Calabrese, J. Gopalakrishnan, E. M. McCarron, K. J. Morrissey, T. R. Askew, R. B. Flippen, U. Chowdhry, A. W. Sleight, Structures of the superconducting oxides $\mathrm{Tl}_{2} \mathrm{Ba}_{2} \mathrm{CuO}_{6}$ and $\mathrm{Bi}_{2} \mathrm{Sr}_{2} \mathrm{CuO}_{6}$, Phys. Rev. B 38 (1988) 225-231. doi:10.1103/PhysRevB.38.225

[19] Y. Shimakawa, Y. Kubo, T. Manako, H. Igarashi, Variation in $T_{c}$ and carrier concentration in Tl-based superconductors, Phys. Rev. B 40 (1989) 11400-11402. doi:10.1103/PhysRevB.40.11400

[20] Y. Shimakawa, Chemical and structural study of tetragonal and orthorhombic $\mathrm{Tl}_{2} \mathrm{Ba}_{2} \mathrm{CuO}_{6}$, Physica C 204 (1993) 247-261. doi: 10.1016/0921-4534(93) 91006-H

[21] C. Ström, S.-G. Eriksson, L.-G. Johansson, A. Simon, H. J. Mattausch, R. K. Kremer, The effect of thallium and oxygen stoichiometry on structure and $T_{c}$ in Tl-2201 and Tl-2212, J. Solid State Chem. 109 (1994) 321-332. doi:10.1006/jssc.1994.1110 
[22] M. A. G. Aranda, D. C. Sinclair, J. P. Attfield, A. P. Mackenzie, Cation distributions and possible phase separation in $\mathrm{TL}_{2} \mathrm{Ba}_{2} \mathrm{CuO}_{6}+\delta$ from synchrotron powder $\mathrm{x}$ ray diffraction, Phys. Rev. B 51 (1995) 12747-12753. doi:10.1103/PhysRevB.51.12747

[23] A. W. Hewat, P. Bordet, J. J. Capponi, C. Chaillout, J. Chenavas, M. Godinho, E. A. Hewat, J. L. Hodeau, M. Marezio, Preparation and neutron diffraction of superconducting "tetragonal" and non-superconducting orthorhombic $\mathrm{Tl}_{2} \mathrm{Ba}_{2} \mathrm{Cu}_{1} \mathrm{O}_{6}$, Physica $\mathrm{C} \quad 156$ (1988) 369-374. doi:10.1016/0921-4534(88)90760-5

[24] G. M. Sheldrick, A short history of SHELX, Acta Cryst. A64 (2007) 112-122. doi:10.1107/S0108767307043930

[25] R. Beyers, S. S. P. Parkin, V. Y. Lee, A. I. Nazzai, R. Savoy, G. Gorman, T. C. Huang, Crystallography and microstructure of Tl-Ca-Ba-Cu-O superconducting oxides, Appl. Phys. Lett. 53 (1988) 432-434. doi:10.1063/1.100611 